\title{
Morphometric analysis of aerobic Eimeria bovis sporogony using live cell 3D holotomographic microscopy imaging
}

\author{
Sara Lopez-Osorio ${ }^{1,2} \cdot$ Zahady D. Velasquez $^{2} \cdot$ Iván Conejeros $^{2} \cdot$ Anja Taubert $^{2} \cdot$ Carlos Hermosilla $^{2}$
}

Received: 8 June 2021 / Accepted: 28 September 2021 / Published online: 11 October 2021

(c) The Author(s) 2021

\begin{abstract}
Monoxenous Eimeria species are widespread enteropathogenic apicomplexan protozoa with a high economic impact on livestock. In cattle, tenacious oocysts shed by E. bovis-infected animals are ubiquitously found and making infection of calves almost inevitable. To become infectious oocysts, exogenous oxygen-dependent E. bovis sporogony must occur leading to the formation of sporulated oocysts containing four sporocysts each harboring two sporozoites. Investigations on sporogony by live cell imaging techniques of ruminant Eimeria species are still absent in literature as commonly used fluorescent dyes do not penetrate resistant oocyst bi-layered wall. Sporogonial oocysts were daily analyzed by a 3D Cell Explorer Nanolive microscope to explore ongoing aerobic-dependent sporogony as close as possible to an in vivo situation. Subsequently, 3D holotomographic images of sporulating E. bovis oocysts were digitally stained based on refractive indices (RI) of oocyst bi-layered wall and sub-compartments of circumplasm using STEVE software (Nanolive), and the cellular morphometric parameters were obtained. Overall, three different E. bovis sporogony phases, each of them divided into two sub-phases, were documented: (i) sporoblast/sporont transformation into sporogonial stages, (ii) cytokinesis followed by nuclear division, and finally (iii) formation of four sporocysts with two fully developed sporozoites. Approximately $60 \%$ of sporulating E. bovis oocysts accomplished aerobic sporogony in a synchronized manner. E. bovis sporogony was delayed (i.e., 6 days) when compared to an in vivo situation where $2-3$ days are required but under optimal environmental conditions. Live cell 3D holotomography analysis might facilitate the evaluation of either novel disinfectants- or anti-coccidial drug-derived effects on ruminant/avian Eimeria sporogony in vitro as discrimination of sporogony degrees based on compactness, and dry mass was here successfully achieved. Main changes were observed in the oocyst area, perimeter, compactness, extent, and granularity suggesting those parameters as an efficient tool for a fast evaluation of the sporulation degree.
\end{abstract}

Keywords Eimeria bovis $\cdot$ Oocyst $\cdot$ Sporogony $\cdot 3$ D holotomographic microscopy $\cdot$ Live cell imaging

Guest Editor: Klaus Brehm

Sara Lopez-Osorio and Zahady D. Velasquez contributed equally to this work.

Zahady D. Velasquez

zahady.velasquez@vetmed.uni-giessen.de

1 CIBAV Research Group, Faculty of Agrarian Sciences, University of Antioquia, Medellín, Colombia

2 Institute of Parasitology, Biomedical Research Center Seltersberg, Justus Liebig University Giessen, Schuberstrasse 81, 35392 Giessen, Germany

\section{Introduction}

The genus Eimeria contains apicomplexan enteropathogenic protozoa with a high economic impact on livestock worldwide. The prevalence of Eimeria infections in cattle is generally high and might reach $100 \%$ in calves (Cornelissen et al. 1995). Despite the dozen Eimeria species described to date, E. bovis is one of the most pathogenic causing severe typhlocolitis characterized by hemorrhagic diarrhea with sometimes fatal outcome in young animals (Stockdale et al. 1981; Daugschies and Najdrowski 2005). All Eimeria species display a monoxenous life cycle composed of two phases, i.e., exogenous and endogenous phases. In the exogenous phase, freshly defecated non-sporulated oocysts are broadly spread into cattle environments; however, they are not still infective until undergoing aerobic sporogony to become infective 
oocysts. The sporulated oocysts within the genus Eimeria contain four sporocysts with two sporozoites in each. The endogenous phase of the life cycle includes numerous asexual merogonies, depending on Eimeria species (i.e., E. bovis developing two merogonies), in specific host cells, and some specific intestine sections, followed by a sexual gamogony leading after syngamy to the formation of non-sporulated oocysts, which are finally shed through feces into the environment (Hammond et al. 1946; Fayer and Hammond 1967).

As for other species, E. bovis oocysts are highly resistant to adverse environmental conditions, such as inadequate moisture, low temperatures, and micro- or anaerobic environments. Optimal conditions for sporogony are temperatures ranging from 15 to $18{ }^{\circ} \mathrm{C}$ and sufficient aeration. Additionally, E. bovis-sporulated oocysts maintain their infectivity for several months and even survive with ease in harsh Scandinavian or Palearctic winters (Svensson et al. 1994; Lassen et al. 2013). Investigations on E. bovis oocyst resistance demonstrated that oocysts incubated in $2 \%$ potassium dichromate solution at $4{ }^{\circ} \mathrm{C}$ were still infective after 4 and a half years and capable of inducing patent infections of experimentally infected calves (Hermosilla, unpublished data).

There are only a few studies in the literature linking bovine Eimeria sporogony with natural oocyst resistance. Accordingly, successful E. bovis sporogony seems to result in oocysts capable of overcoming adverse environmental conditions (Svensson et al., 1994). Conversely, closely related E. zuernii and E. alabamensis oocysts survived much better at sub-zero temperatures as un-sporulated oocyst stages, while $E$. bovis needed to be in a sporulated status to resist very low temperatures (Svensson et al. 1994). Nevertheless, E. alabamensis, E. zuernii, and E. ellipsoidalis, but not $E$. bovis, were able to undergo asexual sporogony after a month at $-18{ }^{\circ} \mathrm{C}$ (Lassen and Seppä-Lassila 2014). Thus, in cases of continuing bovine coccidiosis outbreaks, the management of the herd as well as species identification should be critically assessed, particularly with respect to hygiene, feeding, animal density, and floor types to achieve a significant reduction of infective oocysts in stables and premises. A quick method to assess the effects of novel disinfectants or anti-coccidial drug treatments on Eimeria sporogony could be a helpful tool to manage cattle coccidiosis in the future and/or to analyze in detail effectiveness of applied environmental disinfectant treatments on sporogony.

As already stated, structural properties of Eimeria oocysts help them survive long periods of time within harsh environments and which have been associated to the oocyst wall composed of two layers (Ferguson et al. 2003; Mai et al. 2009). The oocyst wall of ruminant Eimeria is composed of two distinct layers: the outer layer (500-200 nm) and the inner layer $(40 \mathrm{~nm})$ which prevent mechanical as well as chemical damage of sporozoites. This is one of the reasons why breaking ruminant Eimeria oocysts under laboratory conditions requires special protocols including use of mechanical disruptive proceedings (e.g., high-speed shaking with glass beads) or enzymatic digestion to disrupt the wall (Nyberg and Hammond 1965; Kowalik and Zahner 1999; Hermosilla et al. 2002). In fact, cleaning of oocysts with bleach, or storage in harsh oxidant medium (i.e., potassium dichromate), showed to not alter their infectivity (Hermosilla et al. 2002). The wall is also impermeable to many water-soluble disinfectants and detergents (Monné and Hönig 1954). Furthermore, this resistant wall structure has clearly interfered with application of molecular tools to analyze intra-oocyst structures in detail, including immunofluorescence, confocal, or electron microscopy analysis. Given that, commonly used fluorescent dyes do not penetrate highly resistant Eimeria oocyst wall and might alter sporogony process. This fact might explain why most ultrastructural aspects and/or fluorescence analyses of bovine Eimeria species have focused so far on endogenous merogony and gamogony stages (Taubert et al. 2008) but with exception of exogenous sporogony (Lassen and Seppä-Lassila 2014).

Therefore, the aim of this study was to investigate for the first time aerobic-dependent sporogony of E. bovis oocysts to be as close as possible to an in vivo situation by using the novel 3D live cell holotomography microscopy based on refractive indexes (RI) of intracellular structures without requiring membrane rupture or oocyst fixation to identify intra-oocyst organelles. The 3D holotomography imaging was performed on external and internal E. bovis oocyst changes during the sporogony process. This novel technique allowed detection of several sporogony phases sub-divided into first, second, and third sporogony phases. Besides, based on the high quality of 3D live cell holotomography images, quantification of metabolic dry material of sporont, sporoblast, sporocyst, and sporozoite stages was possible to achieve, allowing fast and easy discriminating between different sporulation phases. These new data will contribute as a baseline study on exogenous sporogony permitting better understanding of different cytokinetic and metabolic pathways to be involved in E. bovis sporogony, as well as a rapid tool to identify or assess the sporulation degree after exposure to novel disinfectants/drugs or harsh environmental conditions.

\section{Material and methods}

\section{Ethics}

All animal procedures were performed according to the Justus Liebig University Giessen Animal Care Committee guidelines, approved by the Ethic Commission for Experimental Animal Studies of the State of Hesse (Regierungspräsidium Giessen, 
GI 18/ 10 No A37/2011, JLU Giessen-No. 494) and in accordance to current German Animal Protection Laws.

\section{Parasites}

For parasite propagation, two 8-week-old Holstein Friesian calves were kept in autoclaved metabolic cages within a large animal facility unit of the Institute of Parasitology (JLU Giessen), equipped with a laminar flow entrance and restricted access to avoid any bovine Eimeria spp. exposure. After deemed, parasite-free calves were orally infected with $3 \times 10^{4}$ sporulated E. bovis oocysts (strain $\mathrm{H}$ ) suspended in tap water according to Hermosilla et al. (2002). During patency, non-sporulated oocysts were isolated from feces beginning at 18 days p.i. according to Jackson (Jackson 1964). Feces containing oocysts were washed sequentially through a set of three sieves (pore sizes 850,250, and $80 \mu \mathrm{m}$, respectively, Retsch ${ }^{\circledR}$ ) with tap water. Final suspension was left to sediment overnight, and supernatant was discarded. The sediment was mixed 1:1 with saturated sucrose solution $(1.3 \mathrm{~g} / \mathrm{mL})$ to a final density of $1.15 \mathrm{~g} / \mathrm{mL}$. The suspension was transferred into plastic trays $(30 \times 20 \times 5 \mathrm{~cm})$, and the level was horizontally adjusted. Plastic trays were filled to the top and thereafter covered with clean glass plates allowing complete contact of suspension with glass surfaces. Every $4 \mathrm{~h}$, the glasses were carefully removed, and adherent oocysts were washed off with tap water into a container. The remaining suspension in plastic trays was stirred up, and the process was repeated up to six times or until few oocysts were isolated (microscopic examination, less than 5 oocysts per field of view at $\times 20$ magnification). Collected $E$. bovis oocysts were diluted in tap water (1:1) and then centrifuged at $600 \times g$ for $10 \mathrm{~min}$.

\section{Exogenous Eimeria bovis sporogony}

Isolated E. bovis un-sporulated oocysts were re-suspended in $2 \%$ potassium dichromate $\left(\mathrm{K}_{2} \mathrm{Cr}_{2} \mathrm{O}_{7}\right.$; Merck) solution at room temperature (RT) with constant oxygenation as reported elsewhere (Duszynski and Wilber 1997). Every day, fifty oocysts were examined by light microscopy (inverted microscope IX81®, Olympus) to observe changes of bilayered oocyst wall, sporont, sporoblast, Stieda body, and micropyle, as well as sporocysts containing complete sporozoite development. These oocyst structures were used as a guide to evaluate the sporulation process according to previous reports (Berto et al. 2014; Florião et al. 2016).

\section{D holotomographic live cell imaging of Eimeria bovis oocysts}

Oocysts of E. bovis at different sporogony stages were carefully collected using a micropipette and washed twice with distilled water. Isolated oocysts were seeded into a 35-mm imaging dish plate (IBIDI®, Martinsried, Germany) with sterile phosphate buffered saline PBS pH 7.4, and the dish was placed inside a top-stage incubator (IBIDI ${ }$, Martinsried, Germany). Refractive index (RI)-based 3D holotomographic images were obtained by using a 3D Cell ExplorerFluo (Nanolive, Switzerland) microscope equipped with a $\times 60$ magnification $(\lambda=520 \mathrm{~nm}$, sample exposure $0.2 \mathrm{~mW} /$ $\mathrm{mm}^{2}$ ) and a field depth of $30 \mu \mathrm{m}$. Images were captured and analyzed using STEVE® software (Nanolive) to obtain a RI-based z-stack. Several oocysts were acquired in the same field of view, but images were further analyzed one by one to identify the different sporulation stages. Images were composed of 96 slices, but only those whose whole oocyst structure was captured were chosen for further analysis and final pictures. All slices chosen are displayed as maximum z-projections, and gamma, brightness, and contrast were adjusted (identically for compared image sets) using Fiji ${ }^{\circledR}$ software (Schindelin et al. 2012).

\section{Statistical analysis}

All presented data were expressed as mean \pm SEM. Data were analyzed by Kruskal-Wallis using GraphPad Prism®9 software. Differences between conditions were estimated by a non-parametric one-way ANOVA multiple comparison test, applying a significance level of 5\% $(\alpha \leq 0.05)$.

\section{Results}

\section{Eimeria bovis sporogony results in six internal oocyst structures before sporozoite release}

From the total amount of 50 oocysts, $40.3 \pm 2.62$ E. bovis oocysts completed sporogony (i.e., containing four sporocysts each of them with two fully developed sporozoites) within 6 days in 2\% potassium dichromate incubation at RT. $E$. bovis oocysts with delayed sporogony needed approximately 9-10 days to reach mature sporocyst formation. Percentages of oocysts without sporogony were less than $10 \%$. In order to identify whether E. bovis-sporulated oocysts contained vital sporozoites, oocysts were submitted to an excystation protocol in vitro. Free-released sporozoites were then used to assess host cell invasion and further merogony development in primary bovine umbilical vein endothelial cells (BUVEC) in vitro. As such, extracellular sporozoite gliding motility, sporozoite host cell invasion, and trophozoite and macromeront formation with production of viable merozoites I were evaluated (Hermosilla et al. 2002, 2015; López-Osorio et al. 2020).

The in vitro sporogony was evaluated by a new 3D holotomography microscopy technology, in order to identify in 
detail membrane composition and cytoplasmic contents of diverse organelles without fixation or staining steps. In order to avoid background noise from medium detritus, the samples were washed in PBS before seeding them in a $35-\mathrm{mm}$ imaging dish with a polymer coverslip bottom for high-end microscopy analysis.

For initial analysis, each image was acquired containing 90 slices, and then only the slices that contain the whole oocyst were selected and processed by a maximum z-projection algorithm. This process allowed us to identify the oocyst structure, inner volume, and dry mass of intracellular structures. The 3D rendering permits us to identify in detail the RI density of intra-oocyst structures. Our results show that the intracellular structures are better observed by using the RI density processing than the digital staining method available in the software. The acquisitions show a mix of different oocyst structures and maturation degrees suggesting an unsynchronized sporulation process. The analysis of examined oocysts unveils granules and/or extracellular vesicles contained within the circumplasm, the inner and outer oocyst membranes, the micropyle, the polar granule, the sporont, the sporoblast, the sporocyst membrane, and the fully developed sporozoites containing marked refractile bodies.

Freshly isolated E. bovis oocysts from feces had a large zygote being in close contact with the oocyst bi-layered wall containing several cytoplasmic granules homogeneously distributed in all un-sporulated oocysts (Fig. 1: early contraction phase). At this stage, both the inner and outer oocyst membranes became visible (Fig. 1, bi-layer membrane, yellow arrow). The first sporogonial stage began with the late cytoplasmic contraction phase (CCP), in which the cytoplasmic content is restricted to half volume of the oocyst (Fig. 1). At this early sporogony phase, the

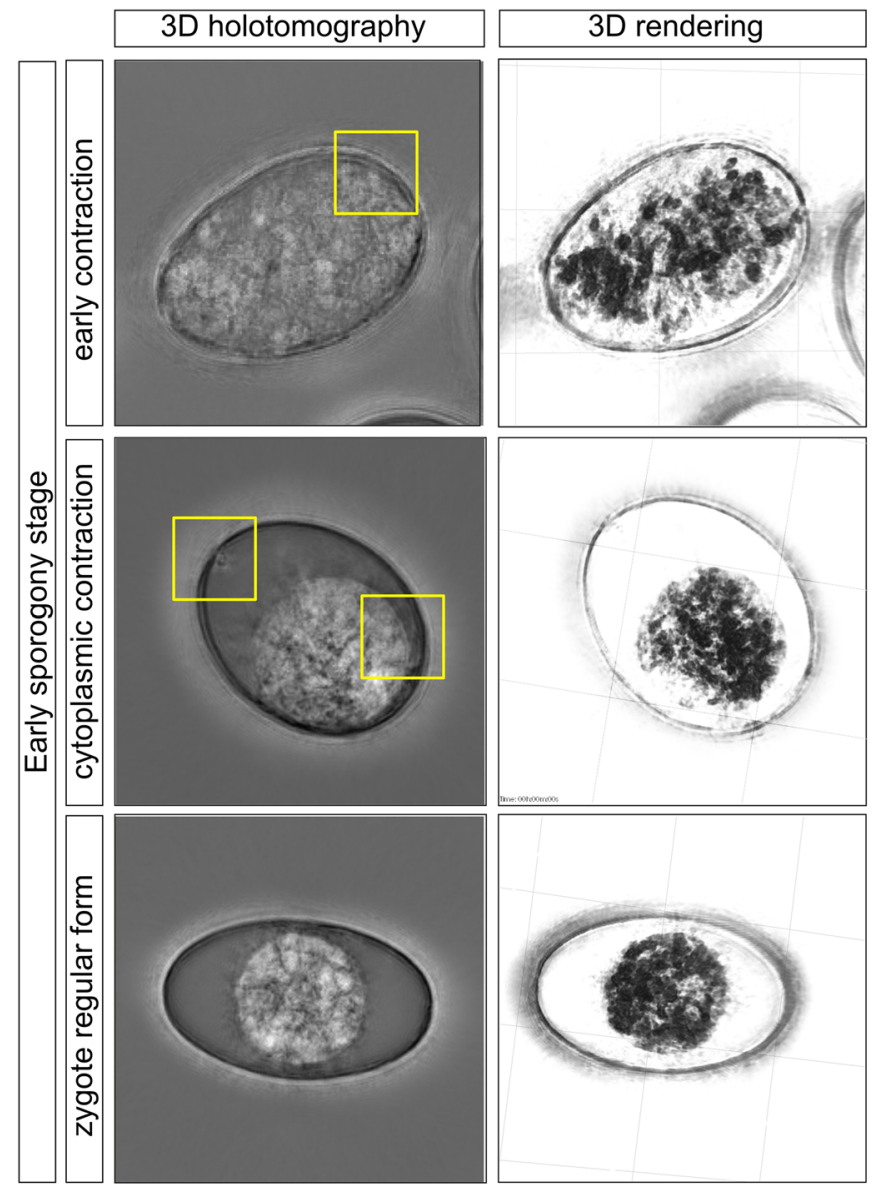

Fig. 1 Early sporogony stage of E. bovis. First sporogony stage in E. bovis oocyst began with a diffuse zygote closely related with the inner oocyst wall (early contraction). Magnification of oocyst wall in contact with the zygote is shown in the $3 \mathrm{D}$ rendering image. The digital staining shows homogeneous distribution of the granules across the circumplasm (purple). After the cytoplasmic contraction, the zygote becomes rounded and placed in the center of the oocyst
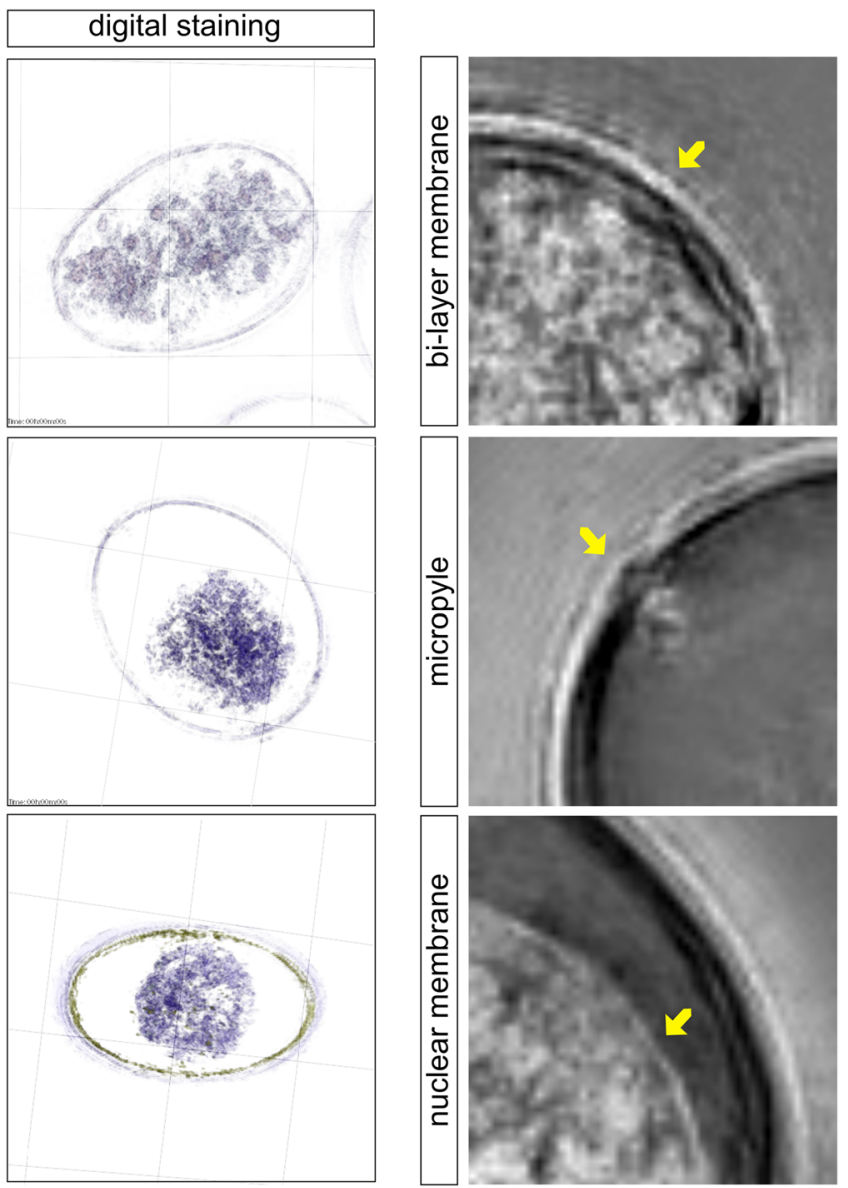

(regular form). The nucleus can be seen as a pale spot in the center of the mass. Closely related with the inner wall and the zygote, some vesicles were seen with a different refractive index (yellow). Images were analyzed using STEVE® software (Nanolive) to obtain a refractive index-based $z$-stack (3D holotomography), the rendering format, and the digital staining based on the refractory index 
micropyle was identified as well as the nuclear membrane (Fig. 1, yellow arrows). The early sporogony stage (ESS) can be sub-divided into three phases, i.e., early CCP, late $\mathrm{CCP}$, and the zygote regular form (ZRF) phases. The total time of ESS in E. bovis was between 24 and $48 \mathrm{~h}$, and the late CCP occurred after oocysts have been suspended in $2 \%$ potassium dichromate (5 oocysts from 50 were sporulated). The zygote shapes changed from circular to oval, and the nucleus was located in a central position of oocysts undergoing sporogony, as a clear spot inside the cytoplasmic mass (Fig. 1, zygote regular form). The size of the sporont at this time point was $17.32 \pm 0.59 \mu \mathrm{m}$ in diameter $(n=10)$.

The second phase of E. bovis sporogony displayed three main stages, i.e., the second nuclear division (SND) and the mid- and late cytokinesis. The SND was only observed $48 \mathrm{~h}$ post incubation in potassium dichromate solution. The SND phase was completed at $72 \mathrm{~h}$ post incubation (Fig. 2).
The SND was observed as a cytoplasmic decompression, and the nuclear membrane was practically absent (Fig. 2, SND). Alongside, the oocyst shape changed from an initial oval form into a circular form. During the second phase of $E$. bovis sporogony, the cytoplasm was gradually reduced, and three/four nuclei per oocysts surrounded by a thin membrane became visible (Fig. 2, yellow arrow in a nuclear membrane image). The bi-layer wall seemed to be fused in some parts of analyzed oocysts and has been observed as a single membrane (Fig. 2, yellow arrow in monolayer membrane). Moreover, at this point, a balloon-like structure was unveiled at the apical zone of oocysts, which corresponded well to the polar granule localization. In this process, $E$. bovis sporonts measured $21 \times 18.9 \mu \mathrm{m}(n=10)$ and were gradually transformed into 4 sporoblasts $(9.95 \times 7.88 \mu \mathrm{m} ; n=10)$ by progressive constriction of their bodies until cleavage was completed (Fig. 3, late cytokinesis). The summary of the total

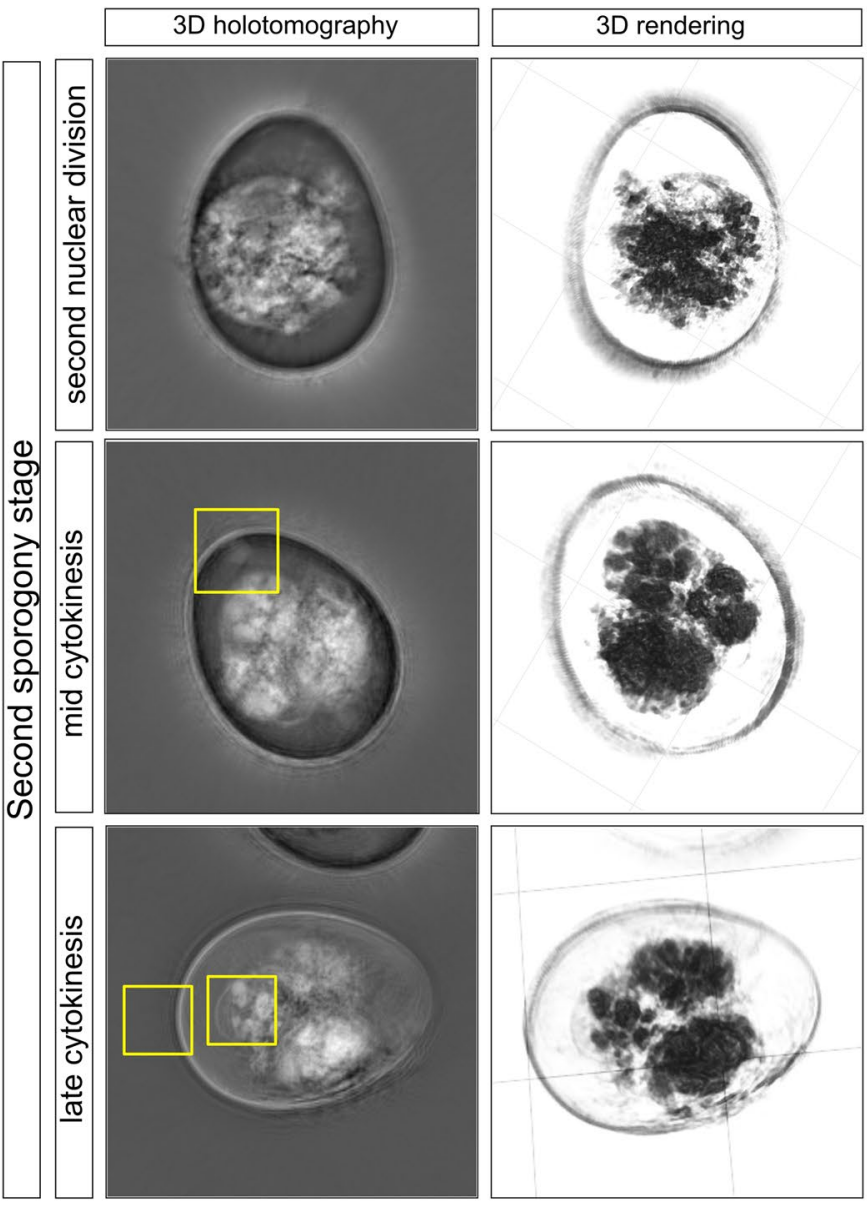

Fig. 2 Second sporogony stage of E. bovis. The nuclear division took place in this stage. The pale spot is no longer in the center of the mass. The nucleus is divided twice, to form 4 nuclei, which can be located in the periphery of the mass. Closely related with the inner wall and the zygote, some vesicles were seen with a different refractive index (yellow square). Cytokinesis begins with the early forma-
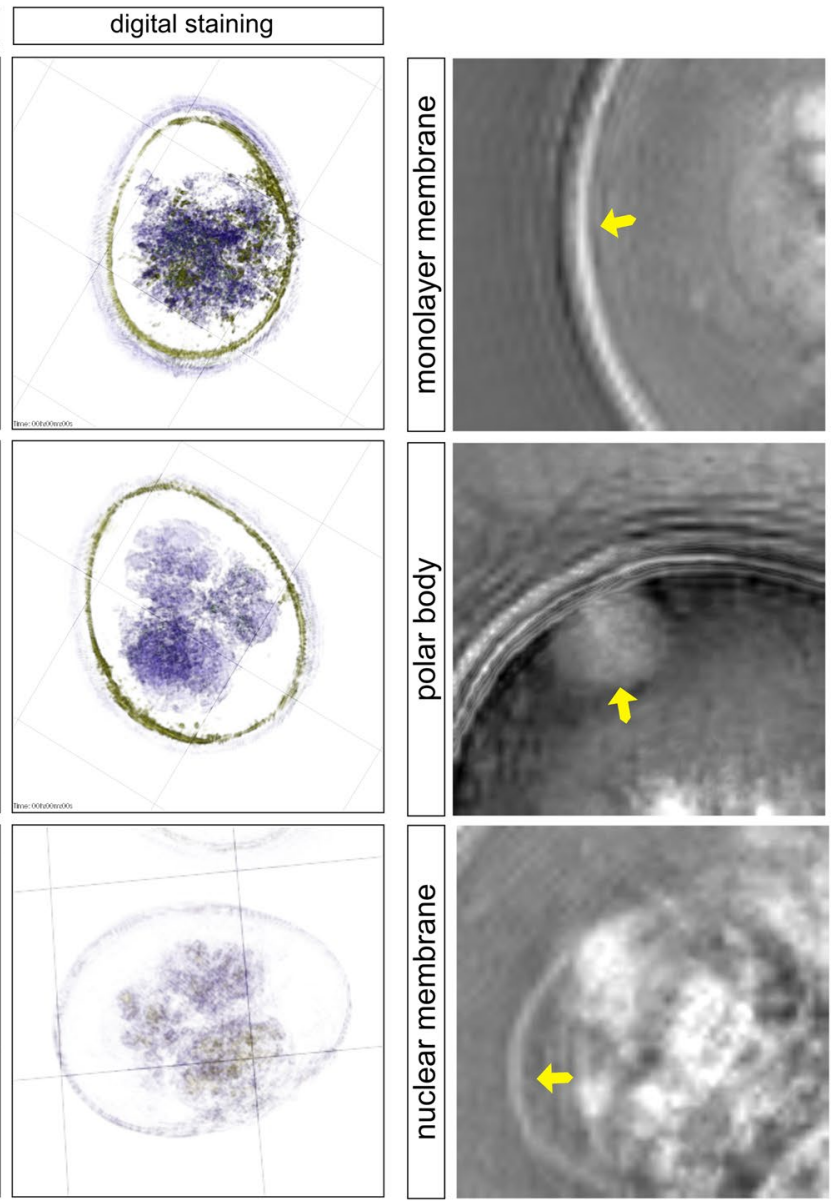

tion of the four sporoblasts (early cytokinesis). Four protrusions, located in perpendicular directions, can be seen in the surface of the zygote (late cytokinesis). Images were analyzed using STEVE® software (Nanolive) to obtain a refractive index-based z-stack (3D holotomography), the rendering format, and the digital staining based on the refractory index 


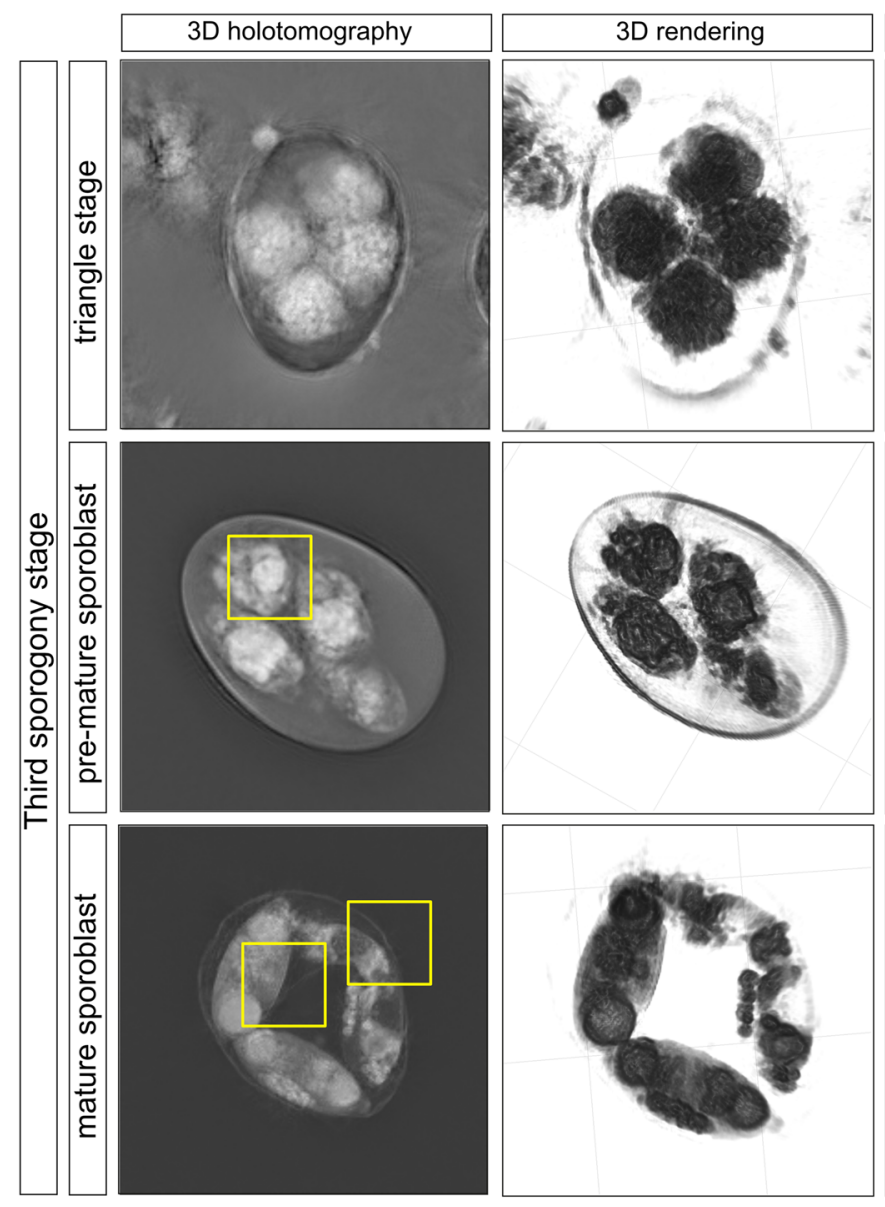

Fig. 3 Late sporogony stage of E. bovis. The protrusions from the late cytokinesis become more prominent, increase in size, and become spherical, forming a clover-shaped structure, until they separate from each other forming the four sporoblasts. The sporoblast becomes elongated and forms a cigar-like shape. Each sporoblast contains granular mass and vacuoles, which develop into the residual body of the sporocyst. The sporoblast continued to become into an ovalshaped sporocyst. Here, a single division of the nuclei occurred, and then a division of the cytoplasm, which results in the formation of

number of oocysts that we recorded in each sporulation step is shown in Table 1.

The third sporogony stage runs with three main steps, triangle, pre-mature, and mature sporoblast stages. Here, the
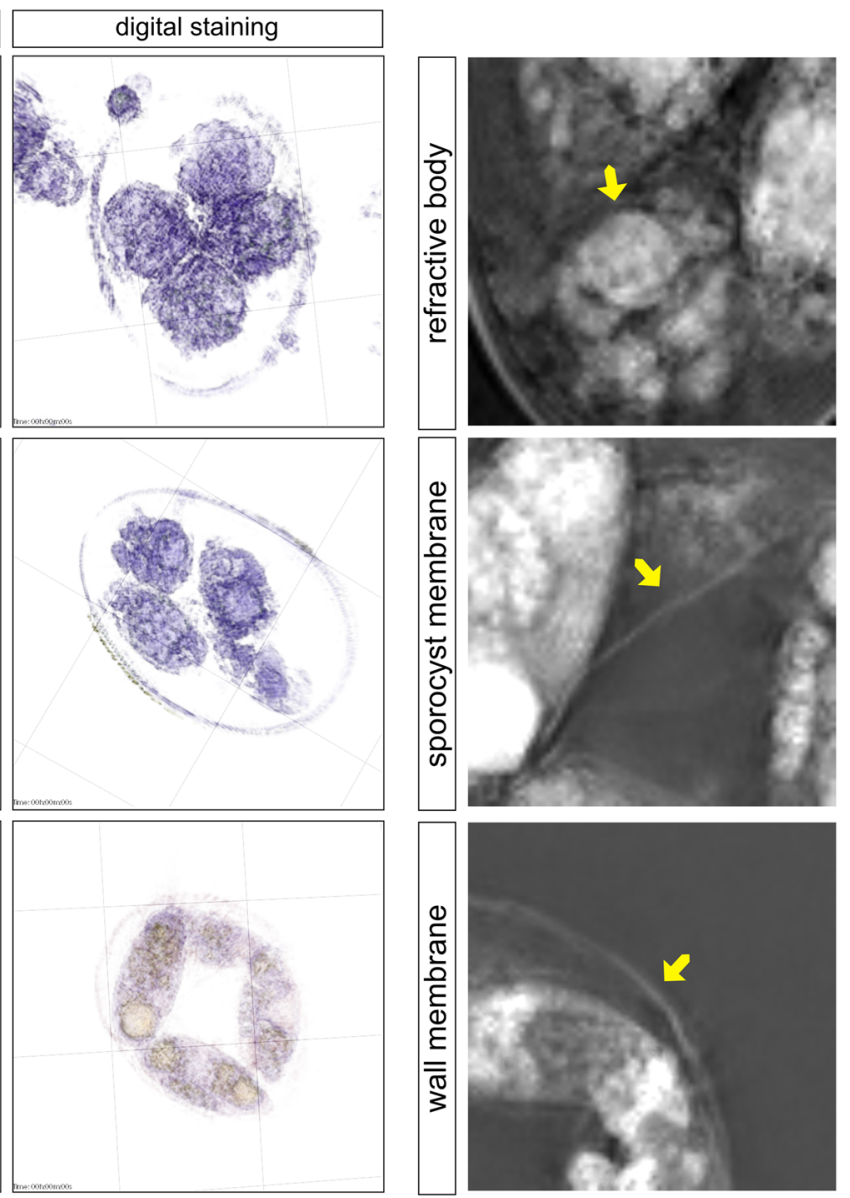

two sporozoites, each one with a visible big refractile body, and the residual body (light-refracting granules). Stieda bodies can be seen as a white tiny lid in one side of the sporocyst (mature oocyst). After the Stieda body and refractile body of the sporozoites are visible, the oocyst is considered mature. Images were analyzed using STEVE® software (Nanolive) to obtain a refractive index-based z-stack (3D holotomography), the rendering format, and the digital staining based on the refractory index

four nucleus divisions became evident as well as first development of two immature sporozoites enclosed within sporoblasts (Fig. 3). Newly formed sporoblasts became rounded and obtained a clover-like shape. After this, sporoblasts

Table 1 E. bovis sporogony stage quantification

\begin{tabular}{|c|c|c|c|c|c|}
\hline \multicolumn{6}{|l|}{ Sporogony* stages } \\
\hline \multicolumn{2}{|l|}{ Early } & \multicolumn{2}{|l|}{ Second } & \multicolumn{2}{|l|}{ Third } \\
\hline Stage & Number & Stage & Number & Stage & Number \\
\hline Zygote regular form & 18 & Late cytokinesis & 2 & Mature sporoblast & 2 \\
\hline Cytoplasmic contraction & 6 & Mid cytokinesis & 5 & Pre-mature sporoblast & 2 \\
\hline Early contraction & 7 & Second nuclear division & 6 & Triangle stage & 2 \\
\hline
\end{tabular}

*Fifty oocysts were counted and classified according to the different sporogony stages they were 
began to elongate forming the cigar-shaped sporoblasts $(12.78 \pm 1.3 \times 6.19 \pm 0.72 \mu \mathrm{m})$. During the third sporogony stage, also refractive bodies (RF) of sporozoites became visible for the first time (Fig. 3, yellow arrow). After RF formation, the Stieda body and previously formed RF were visible in newly formed sporozoite stages within four sporocysts (Fig. 4B). Sporocysts were considered fully mature at $96-120 \mathrm{~h}$ of incubation containing two well-developed sporozoites with clear anterior and posterior RF formation. At the end of sporogony (final sporogonial phase), residual material inside sporocysts $(14.7 \pm 2.22 \times 6.5 \pm 0.58 \mu \mathrm{m})$ was clearly visible as a cumulus of extracellular vesicles (Fig. 4C, yellow arrow). The same holds true for the oocyst circumplasm containing as well residual material collected in multiple granules or vesicles of different sizes. The sporocysts of E. bovis are ovoid and contain two sporozoites (Fig. 4D). Each sporozoite showed a large RF in the base opposite to their partner. The Stieda bodies (SB) were located at the narrow end of sporocysts. These SB were visible as plugs covering the sporocyst micropyle. This area represents the egress for sporozoites from sporocysts. During E. bovis excystation, circumplasmic granules were released in a fast-sorted way from opened micropyle. Interestingly, circumplasmic granules often surrounded free-released and highly motile sporozoites and kept in close contact to the sporozoite surface, while these infective stages were moving away from empty oocysts.

\section{Morphometric analysis of $\mathrm{E}$. bovis oocyst structures as a useful tool for sporogony stage discrimination}

Eimeria spp. oocyst sporulation in the environment is conditioned by many factors including humidity, oxygen pressure, or temperature, which all can hamper production of infective units, namely, sporulated oocysts. However, the degree of oocyst sporulation is quite difficult to achieve in vitro. 3D
Fig. 4 Eimeria bovis oocyst. Non-sporulated (A) and sporulated (B) oocysts. The four sporocysts of E. bovis sporulated oocysts are ovoid and contain two sporozoites. The average length was $14.7 \pm 2.22 \mu \mathrm{m}$ and width $6.5 \pm 0.58$. Normally, the length and width of the sporocyst are one-fourth and one to half of the oocyst. Each sporozoite showed a big refractile body in the base opposite to their partner. C Free sporozoites and residual material inside the sporocyst were clearly visible as a cumulus of extracellular vesicles (yellow arrow). The Stieda bodies were located in the narrowed end of the sporocyst. These are plugs which are covering the sporocyst micropyle. D Sporocyst
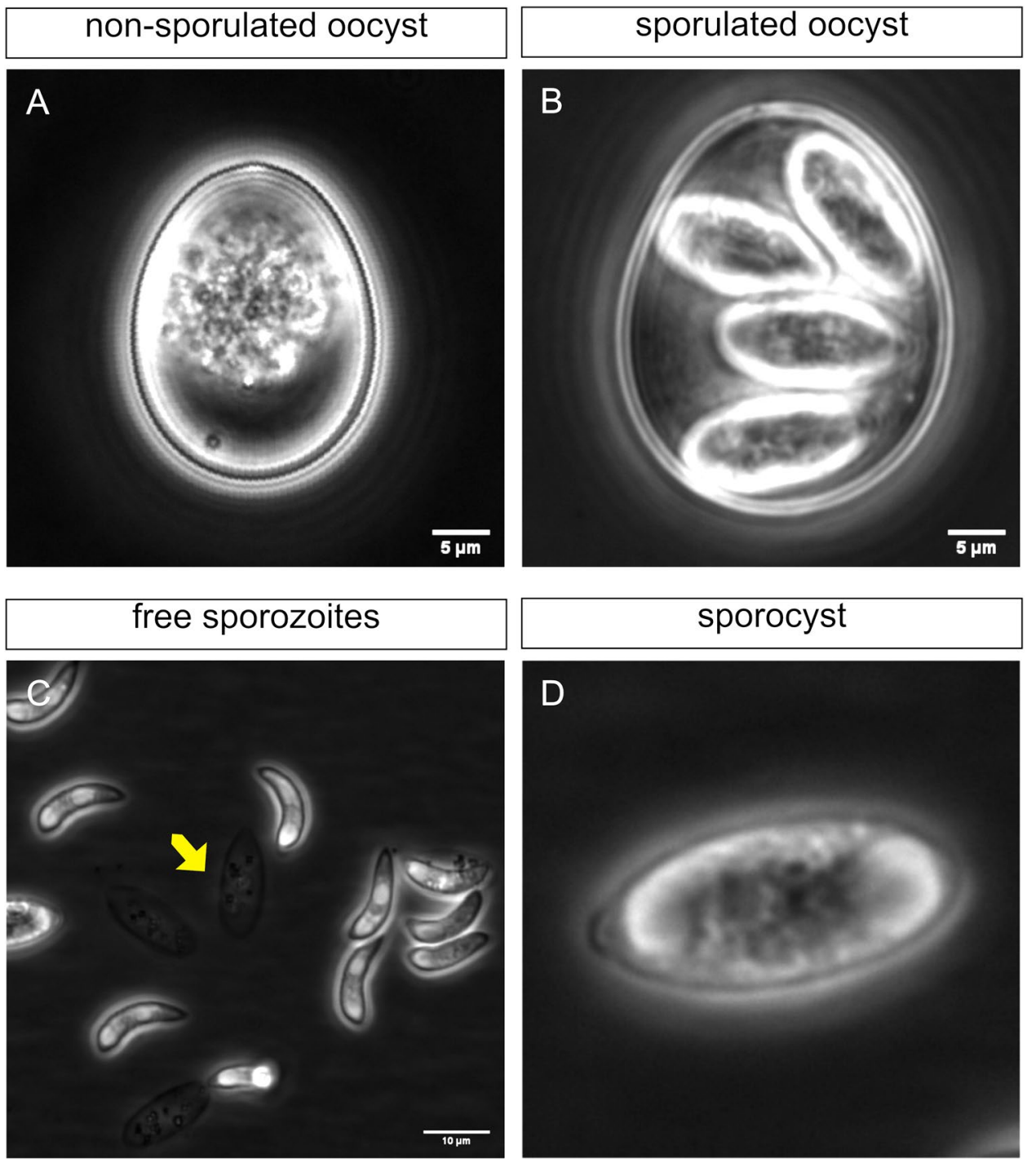
holotomography microscopy analysis could be a powerful tool to evaluate this process in order to assess, for example, the effectiveness of a treatment against this infective stage. By using 3D-generated images, all sporulation stages were clearly identified, and by using additional image analysis tools, we measured several oocyst parameters. A CellProfiler pipeline was designed to load each 2D RI map; segment the contained oocysts using the primary objects detection module; and extract area, shape, and intensity features using the measurements modules. A critical point for proper oocyst detection was to use a correct threshold value, while automatic threshold detection is suited for most fluorescent microscopy images, whose signal dynamics are related to a different type of factors. In contrast, the RI images are quantitative and depend only on the nature of the biological object that is examined. Therefore, the threshold can be entered as a fixed value, ensuring the same analytical conditions in all the samples. The oocyst sizes were considered to encompass the full spectrum of potential diameters. Once segmented, these objects were finally used to extract the area, perimeter, dry mass, compactness, extent, and granularity in each frame of the time-lapse experiment. The data were exported as a.csv file. The results showed that the area of the oocyst was dramatically increased in the second sporogony stage, but it was not different between the first and third stages (Fig. 5). Nevertheless, the oocyst perimeter decreased according to the sporogony stages proceeded (Fig. 5). Two parameters that combined allowed a good discrimination of sporogony degree were compactness and dry mass, which were significantly increased and decreased when oocysts reached the last sporogony phase (Fig. 5). Finally, results on granularity of E. bovis oocysts clearly showed that oocysts in the third sporogonial stage displayed a reduction in granularity when compared with the previous two sporogonial phases.

\section{Discussion}

Complete life cycle of $E$. bovis has been well documented in the past except for the exogenous sporogony generation (Hammond et al. 1946; Fayer and Hammond 1967). Asexual E. bovis oocyst sporogony is tightly regulated and complex and represents a necessary step for becoming infective stages, namely, a sporulated oocyst. Although these facts, E. bovis sporogony, as well as other ruminant species, has infrequently been explored in the past. To our best knowledge, this work represents first documentation of whole exogenous $E$. bovis sporogony using a novel live cell imaging 3D holotomographic microscopy analysis in vivo without fixation or interference through staining procedures.

Exogenous sporogony is an oxygen-dependent metabolic dividing process, and consequently Senger demonstrated that at least an oxygen tension of $15 \mathrm{~mm}$ of $\mathrm{Hg}$ was necessary for completing this parasitic replication (Senger 1959). Additionally, Eimeria oocysts also require suitable temperatures and relative humidity for fulfilling exogenous sporogony. Previous reports described complete E. bovis sporogony naturally occurring within 2-3 days under optimal temperature, oxygen, and humidity conditions, but in our study, E. bovis sporogony showed to be longer by 2-3 days. In the present study, $90 \%$ of oocyst sporulation rates were achieved after 6 days at constant RT $\left(24-25^{\circ} \mathrm{C}\right)$ and oxygenation, whereas in a former study, E. bovis oocysts were kept at constant $28{ }^{\circ} \mathrm{C}$ and needing 3 days for $90 \%$ sporulation (Pyziel and Demiaszkiewicz 2015). These results are consistent with observations of many authors with respect to the influence of temperature on Eimeria spp. sporogony. Just by one degree Celsius lower, namely $23{ }^{\circ} \mathrm{C}$, under natural conditions, $E$. bovis sporogony was delayed by up to 25 days (Wagenbach and Burns 1969), and at much lower temperatures $\left(3-5{ }^{\circ} \mathrm{C}\right)$ sporogony was prolonged for up to100 days demonstrating temperature-derived effects on exogenous Eimeria asexual replication (Pyziel and Demiaszkiewicz 2015). Most authors agreed that the use of anti-bacterial or anti-fungal agents (i.e., potassium dichromate) as oocyst conservation solution helped achieve higher sporogony rates under laboratory conditions (Lotze and Leek 1961; Graat et al. 1994).

Three main stages can be differentiated in E. bovis sporogony: (i) early sporogony stage (sub-divided in un-sporulated oocyst stage, less concentrated sporoblasts, and concentrated sporoblasts), (ii) mid sporogony stage (sub-divided in nuclear division and cytokinesis), and (iii) late sporogony stage (four rounded sporoblasts and mature sporoblasts). These sporogonial stages have been reported for other nonruminant Eimeria species, such as chicken E. maxima and $E$. tenella (Wagenbach and Burns 1969). Nonetheless, for these two avian species, the classical pyramid-shaped sporoblasts were documented after $24 \mathrm{~h}$ of sporogony, which in the case of $E$. bovis oocysts were not seen. Instead, a clover-shaped sporoblast form was detected during aerobic $E$. bovis sporogony. Morphological differences of eimerian sporoblasts might result from the fact that $E$. bovis oocysts were kept in constant suspension conditions while performing asexual cellular division. Conversely, other sporogony-related studies used oocysts added to glass slides and covered with coverslips as performed in all avian Eimeria sporogony-related studies, which might have physically distorted forces on newly formed sporoblasts thereby shaping them differently from $E$. bovis sporoblasts. Interestingly, a similar sporoblast shape was previously described for Cystoisospora felis (former Isospora felis) sporogony. It shows two spherical sporoblast nuclei with central or peripheral localization, similar as we described here for E. bovis sporoblasts (Shah 1970).

After being excreted, the zygote cytoplasm starts to shrink within bi-layered E. bovis oocysts, thereby becoming a more 
Fig. 5 Morphometric characterization of the different stages observed during $E$. bovis sporulation using 3D microscopy. All oocyst stages recorded analyzed based on their RI in order to extract the area, perimeter, dry mass, compactness, extent, and granularity in each frame of the time-lapse experiment. The results show changes in the area, perimeter, compactness, extent, and granularity. The dry mass was decreased with time of maturation. Values are expressed as mean $\pm \mathrm{SEM}$. The value of $p$ is informed in each graph
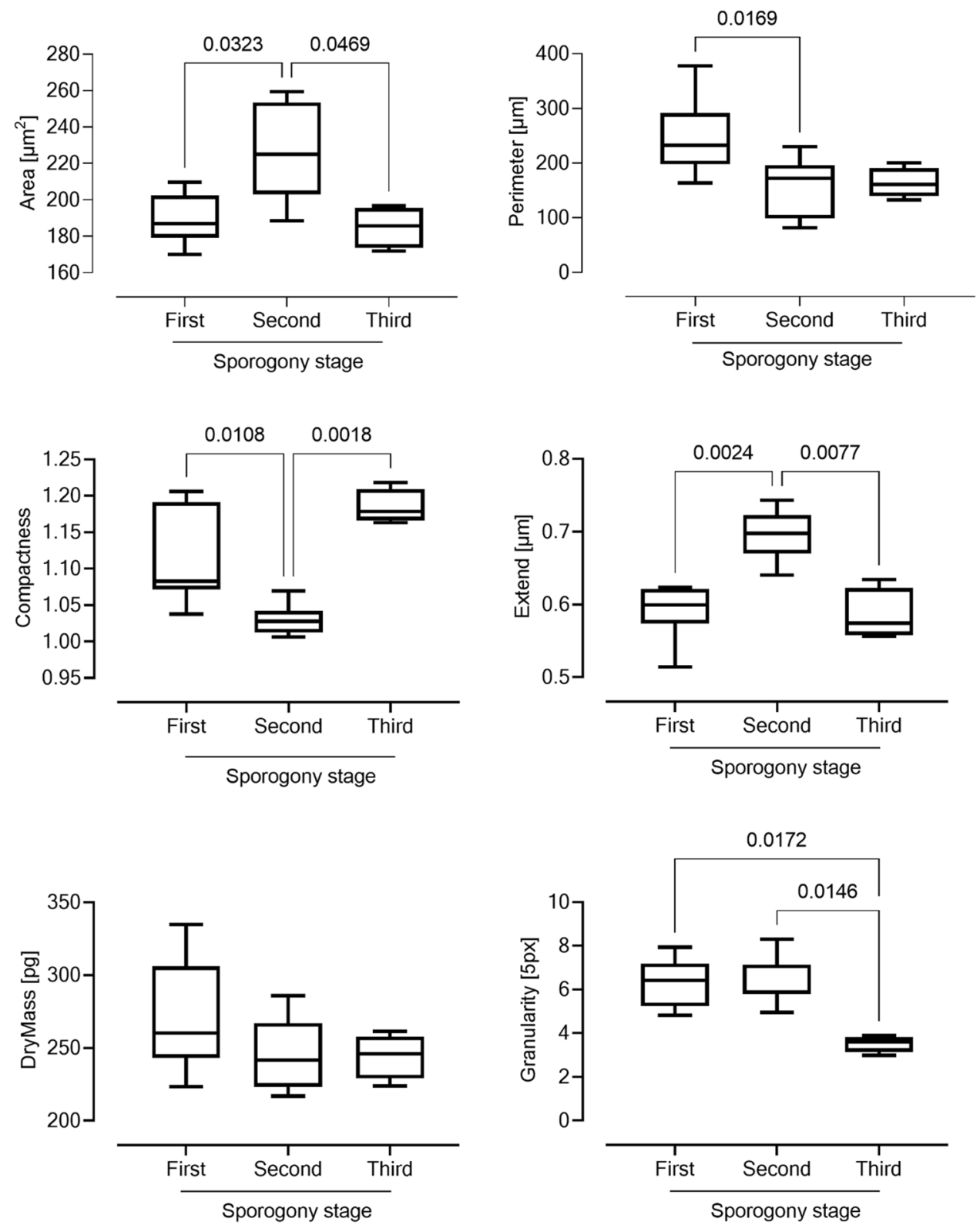

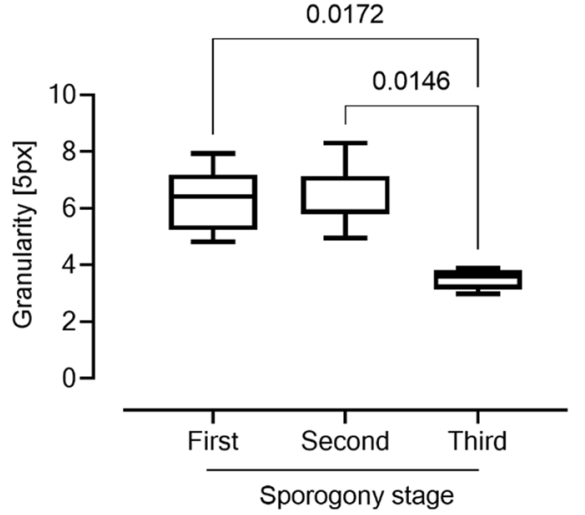

spherical structure. It has been postulated that this zygote shrinkage is associated with oocyst dehydration. After oocyst dehydration, the remaining fluid between the zygote and the oocyst bi-layered wall, known as circumplasm, starts to contain small refracting bodies and bacteria (Yakimov et al. 1940). Exogenous oocyst dehydration has already been reported for a majority of coccidian parasites, with exception of the genera Sarcocystis and Cryptosporidium, both of which undergo endogenous sporogony within the host intestine under anaerobic conditions (Yakimov et al. 1940). In addition, some fish Eimeria species, i.e., E. carpelli and E. subepithelialis, are eliminated from infected host as fully sporulated oocysts (Yakimov et al. 1940).

The molecular content and precise function of granules in oocyst circumplasm are still unknown. During initial sporogony process of E. bovis, we detected via 3D holotomography analysis tiny structures and granules with different RI closely related to the oocyst wall and to the zygote components during sporulation. These spherical structures or granules then disappeared when zygote became a sporoblast. Structures with the same RI were found later on in free-released sporozoites surrounding their prominent apical and basal refractile bodies. More importantly, small granules/vesicles were additionally released while sporozoite egress from oocyst and occurring in a clearly sorted manner. These granules were in close contact to sporozoite surface and be found later on surrounding free-released and motile sporozoites. These circumplasm structures might represent functional extracellular vesicles $(\mathrm{EV})$, microsomes, or exosomes which might influence sporozoite environment 
or modulate host cell innate immune reactions before cell invasion, but further investigation will be needed to clarify this assumption.

The awareness of E. bovis coccidiosis among farmers worldwide, particularly of dairy cattle, has significantly increased in the past decades, therefore raising further questions related to the control of highly resistant environmental oocysts and requesting disinfection strategies. Thus, we call for additional sporogony-related studies in order to gain new data on ruminant Eimeria spp. aerobic-dependent metabolic pathways leading to sufficient energy, blocking units and nutrients while achieving exogenous sporozoite development. Finally, the better understanding of metabolic pathways during aerobic Eimeria spp. sporogony might help identify new disinfection products or even drugs capable of interfering with this exogenous asexual parasite replication and impeding new animal infections.

Acknowledgements The authors wish to acknowledge all staff members of the Institute of Parasitology (JLU, Giessen, Germany) for helping in the maintenance of experimentally E. bovis-infected calves and farther for their excellent collaboration in oocyst isolation. We thank the team of Nanolive SA and especially Marlies Mürnseer for the use of their Open-Source Analysis pipelines applied on our 3D Cell explorer datasets in order to extract and provide quantitative measurements. We also would like to thank the CODI strategy program for supporting research groups in the process of consolidation 2018-2019 of the University of Antioquia, Colombia. Dr Velásquez would like to thank Büro für Chancengleichheit of the Justus-Liebig-Universität Gießen for the funds assigned to help her research.

Author contribution SLO: sporulation protocol and sample preparation. ZDV and IC: image's acquisition. ZDV: manuscript figures preparation and Nanolive images analysis and quantification. SLO and ZDV: manuscript writing. IC, AT, and $\mathrm{CH}$ : manuscript drafting and editing. $\mathrm{CH}$ and AT coordinated the Eimeria bovis project. All the authors have read and approved the manuscript as submitted.

Funding Open Access funding enabled and organized by Projekt DEAL. This study was funded by the Institute of Parasitology, Faculty of Veterinary Medicine of the Justus Liebig University Giessen (JLU), Giessen, Germany. SLO was a recipient of a PhD fellowship financed by the German Exchange Academic Service (DAAD).

Open Access This article is licensed under a Creative Commons Attribution 4.0 International License, which permits use, sharing, adaptation, distribution and reproduction in any medium or format, as long as you give appropriate credit to the original author(s) and the source, provide a link to the Creative Commons licence, and indicate if changes were made. The images or other third party material in this article are included in the article's Creative Commons licence, unless indicated otherwise in a credit line to the material. If material is not included in the article's Creative Commons licence and your intended use is not permitted by statutory regulation or exceeds the permitted use, you will need to obtain permission directly from the copyright holder. To view a copy of this licence, visit http://creativecommons.org/licenses/by/4.0/.

\section{References}

Berto BP, McIntosh D, Lopes CWG (2014) Studies on coccidian oocysts (Apicomplexa: Eucoccidiorida). Rev Bras Parasitol Vet 23:1-15. https://doi.org/10.1590/S1984-29612014001

Cornelissen AWCA, Verstegen R, van den Brand H, Perie NM, Eysker M, Lam TJGM et al (1995) An observational study of Eimeria species in housed cattle on Dutch dairy farms. Vet Parasitol 56:716. https://doi.org/10.1016/0304-4017(94)00671-X

Daugschies A, Najdrowski M (2005) Eimeriosis in cattle: current understanding. J. Vet. Med B Infect Dis Vet Public Health 52:417427. https://doi.org/10.1111/j.1439-0450.2005.00894.x

Duszynski D, Wilber P (1997) A guideline for the preparation of species descriptions in the Eimeriidae. Faculty Publications from the Harold W. Manter Laboratory of Parasitology. Available at: https://digitalcommons.unl.edu/parasitologyfacpubs/156

Fayer R, Hammond DM (1967) Development of first-generation schizonts of Eimeria bovis in cultured bovine cells. J Protozool 14:764-772

Ferguson DJP, Belli SI, Smith NC, Wallach MG (2003) The development of the macrogamete and oocyst wall in Eimeria maxima: immuno-light and electron microscopy. Int J Parasitol 33:13291340. https://doi.org/10.1016/S0020-7519(03)00185-1

Florião MM, Lopes B, do B., Berto, B. P., and Lopes, C. W. G. (2016) New approaches for morphological diagnosis of bovine Eimeria species: a study on a subtropical organic dairy farm in Brazil. Trop Anim Health Prod 48:577-584. https://doi.org/10.1007/ s11250-016-0998-5

Graat EA, Henken AM, Ploeger HW, Noordhuizen JP, Vertommen MH (1994) Rate and course of sporulation of oocysts of Eimeria acervulina under different environmental conditions. Parasitology 108(Pt 5):497-502. https://doi.org/10.1017/s0031182000077350

Hammond DM, Bowman GW, Davis LR, Simms BT (1946) The endogenous phase of the life cycle of Eimeria bovis. J Parasitol 32:409-427. https://doi.org/10.2307/3272876

Hermosilla C, Barbisch B, Heise A, Kowalik S, Zahner H (2002) Development of Eimeria bovis in vitro: suitability of several bovine, human and porcine endothelial cell lines, bovine fetal gastrointestinal, Madin-Darby bovine kidney (MDBK) and African green monkey kidney (VERO) cells. Parasitol Res 88:301-307

Hermosilla C, Stamm I, Menge C, Taubert A (2015) Suitable in vitro culture of Eimeria bovis meront II stages in bovine colonic epithelial cells and parasite-induced upregulation of CXCL10 and GM-CSF gene transcription. Parasitol Res 114(8):3125-3136. https://doi.org/10.1007/s00436-015-4531-y

Jackson AR (1964) The isolation of viable coccidial sporozoites. Parasitology 54:87-93

Kowalik S, Zahner H (1999) Eimeria separata: method for the excystation of sporozoites. Parasitol Res 85:496-499. https://doi.org/10. $1007 / \mathrm{s} 004360050584$

Lassen B, Seppä-Lassila L (2014) Recovery and sporulation of bovine Eimeria oocysts after exposure to sub-zero temperature. Veterinarija Ir Zootechnika 66:35-39

Lassen B, Lepik T, Bangoura B (2013) Persistence of Eimeria bovis in soil. Parasitol Res 112:2481-2486. https://doi.org/10.1007/ s00436-013-3413-4

López-Osorio S, Silva LMR, Chaparro-Gutierréz JJ, Velásquez ZD, Taubert A, Hermosilla C (2020) Optimized excystation protocol for ruminant Eimeria bovis- and Eimeria arloingi-sporulated oocysts and first 3D holotomographic microscopy analysis of differing sporozoite egress. Parasitol Int 76:102068. https://doi.org/ 10.1016/j.parint.2020.102068

Lotze JC, Leek RG (1961) A practical method for culturing coccidial oocysts in tap water. J Parasitol 47:588-590 
Mai K, Sharman PA, Walker RA, Katrib M, Souza DD, McConville MJ et al (2009) Oocyst wall formation and composition in coccidian parasites. Mem Inst Oswaldo Cruz 104:281-289. https://doi.org/ 10.1590/S0074-02762009000200022

Monné L, Hönig G (1954) On the properties of the shells of coccidian oocysts. Arkiv För Zool 7:251-256

Nyberg PA, Hammond DM (1965) Description of the sporulated oocysts and sporozoites of four species of bovine coccidia. $\mathrm{J}$ Parasitol 51:669-673

Pyziel AM, Demiaszkiewicz AW (2015) Observations on sporulation of Eimeria bovis (Apicomplexa: Eimeriidae) from the European bison Bison bonasus: effect of temperature and potassium dichromate solution. Folia Parasitol 62:20-20. https://doi.org/10.14411/ fp. 2015.020

Schindelin J, Arganda-Carreras I, Frise E, Kaynig V, Longair M, Pietzsch T et al (2012) Fiji: an open-source platform for biologicalimage analysis. Nat Methods 9:676-682. https://doi.org/10.1038/ nmeth.2019

Senger CM (1959) Chemical inhibition of sporulation of Eimeria bovis oocysts. Exp Parasitol 8:244-248. https://doi.org/10.1016/00144894(59)90023-2

Shah HL (1970) Sporogony of the oocysts of Isospora felis Wenyon, 1923 from the Cat*. J Protozool 17:609-614. https://doi.org/10. 1111/j.1550-7408.1970.tb04737.x
Stockdale PH, Bainborough AR, Bailey CB, Niilo L (1981) Some pathophysiological changes associated with infection of Eimeria zuernii in calves. Can J Comp Med 45:34-37

Svensson C, Uggla A, Pehrson B (1994) Eimeria alabamensis infection as a cause of diarrhoea in calves at pasture. Vet Parasitol 53:33-43. https://doi.org/10.1016/0304-4017(94)90014-0

Taubert A, Hermosilla C, Sühwold A, Zahner H (2008) Antigeninduced cytokine production in lymphocytes of Eimeria bovis primary and challenge infected calves. Vet Immunol Immunopathol 126:309-320. https://doi.org/10.1016/j.vetimm.2008.09.003

Wagenbach GE, Burns WC (1969) Structure and respiration of sporulating Eimeria stiedae and E. tenella oocysts. J Protozool 16:257263. https://doi.org/10.1111/j.1550-7408.1969.tb02266.x

Yakimov VL, Senyushkina VP, Machul'skii, S. N. (1940) Coccidiosis of G. pigs in the U.S.S.R. Vestnik Mikrobiologii 17:391-401

Publisher's note Springer Nature remains neutral with regard to jurisdictional claims in published maps and institutional affiliations. 